\title{
A NEW PROOF FOR BERGWEILER'S CONJECTURE CONCERNING THE FIRST DERIVATIVE OF TRANSCENDENTAL MEROMORPHIC FUNCTION WITH PICARD EXCEPTIONAL VALUE
}

\author{
FENG GUO AND YUHUA LI
}

\begin{abstract}
Let $f$ be a infinite order meromorphic function, suppose $f^{\prime}$ omits the value 1 in $\mathbb{C}$. Then $M_{f}=f^{\prime}\left(f^{-1}(0)\right)$ is unbounded. We give a new proof for the case of infinite order for Bergweiler's conjecture.
\end{abstract}

\section{Introduction and main result}

Let $f$ be a meromorphic function in $\mathbb{C}$, define $M_{f}=f^{\prime}\left(f^{-1}(0)\right)=\left\{f^{\prime}(z): z \in \mathbb{C}\right.$ and $f(z)=$ $0\}$. W.Bergweiler [1] raised a conjecture in 2001:

Conjecture 1: Let $f$ be a transcendental meromorphic function in $\mathbb{C}$. If $f^{\prime}(z) \neq 1$ for all $z \in \mathbb{C}$, then $M_{f}$ is unbounded.

Considering $g(z)=z-f(z)$ we see Conjecture 1 is equivalent to the following one:

Conjecture 2: Let $g$ be a transcendental meromorphic function in $\mathbb{C}$. Suppose that $g^{\prime}$ does not have zeros. Then there exists a sequence $\left\{z_{n}\right\}_{n=1}^{\infty}$ of fixed points of $g$ such that $\left|g^{\prime}\left(z_{n}\right)\right| \rightarrow \infty$.

Bergweiler [1] has separately proved Conjecture 1 is affirmative for finite order meromorphic functions and entire functions; Jianming Chang [2] has first confirmed the conjecture for infinite order meromorphic functions, which is based on the theory of quasinormal families. In this paper, we only use the the tool of normal families to affirm the case.

Theorem 1. Let $f$ be a transcendental meromorphic function. If $f^{\prime}(z) \neq 1$ for all $z \in \mathbb{C}$, then $M_{f}$ is unbounded.

Received March 22, 2014, accepted June 27, 2014.

2010 Mathematics Subject Classification. Primary 30D30; Secondary 30D45,30D35.

Key words and phrases. Transcendental meromorphic function, omit value, derivative.

Corresponding author: Feng Guo.

The second author partially supported by the grant 11261069 from the National Science Foundation of China. 
According to the conjecture, we can see if meromorphic function $f$ satisfies $f^{\prime}(z) \neq 1$ for all $z \in \mathbb{C}, f$ is rational when $M_{f}$ is bounded and $f$ is transcendental when $M_{f}$ is unbounded. Then whether $M_{f}$ is bounded can be an useful divide standard for meromorphic function.

\section{Preliminary lemmas}

Remark: Let $D\left(z_{0}, M\right)=\left\{z:\left|z-z_{0}\right|<M\right\}$ and sign $f_{n}(z) \stackrel{\text { loc. }}{\Longrightarrow} g(z)$ means a sequence of meromorphic functions $\left\{f_{n}(z)\right\}_{n=1}^{\infty}$ locally uniformly convergence to a meromorphic function $g(z)$.

Lemma 1 ([3]). Let $f$ be a meromorphic function. If the spherical derivative $f^{\#}(z)$ of $f(z)$ is bounded. Then the order of $f(z)$ is at most 2 .

Lemma 2 ([4]). Let $\mathscr{F}$ be a family of meromorphic functions in a domain D. Suppose that there exists $K>0$ such that $M_{g} \subset \overline{D(0, K)}$ for all $g \in \mathscr{F}$.If $\mathscr{F}$ is not normal, then there exists a sequence $\left\{f_{n}\right\}_{n=1}^{\infty}$ in $\mathscr{F}$, a sequence $\left\{z_{n}\right\}_{n=1}^{\infty}$ in $D$, a sequence $\left\{\rho_{n}\right\}_{n=1}^{\infty}$ of positive real numbers and a non-constant finite order function $f$ which is meromorphic in $\mathbb{C}$ such that $z_{n} \rightarrow z_{0}$ for some $z_{0} \in D, \rho_{n} \rightarrow 0$ and

$$
\frac{f\left(z_{n}+\rho_{n} z\right)}{\rho_{n}} \stackrel{\text { loc. }}{\Longrightarrow} f(z) \quad(z \in \mathbb{C} ; n \rightarrow \infty) .
$$

Moreover, the spherical derivative $f^{\#}(z)$ off satisfies $f^{\#}(z) \leq f^{\#}(0)=K+1$ for all $z \in \mathbb{C}$.

In the conclusion of Lemma 2 , it is possible to replace $f_{n}\left(z_{n}+\rho_{n} z\right) / \rho_{n}$ by $f_{n}\left(z_{n}+\rho_{n} z\right) / \rho_{n}^{\alpha}$, for any given $\alpha$ satisfying $-1<\alpha \leq 1$, but here the case $\alpha=1$ suffices. The case $\alpha=0$ is due to Zalcman [5], and the case $-1<\alpha<1$ is due to Pang [6,7]. In Lemma 2, if all $g \in \mathscr{F}$ have no zero in $D$, then $M_{g}=\varnothing$. The conclusion is still true according to the original proof in which $K$ can take 0 .

Lemma 3 ([1]). Let $f$ be a meromorphic function in $\mathbb{C}$ with finite order. Suppose $f^{\prime}(z) \neq 1$ for all $z \in \mathbb{C}$ and there exists $K>0$ such that $M_{f} \subset \overline{D(0, K)}$. Then $f$ is rational and has the form

$$
\text { (a) } f(z)=z+a+\frac{b}{(z-c)^{l}} \text { with } a, b, c \in \mathbb{C}, b \neq 0, l \in \mathbb{N}
$$

or the form $(b) f(z)=\alpha z+\beta, \alpha, \beta \in \mathbb{C}, \alpha \neq 1$.

Lemma 4 ([1]). Let $D \in \mathbb{C}$ be a domain and $\mathscr{F}$ be a family of functions meromorphic in $D$, for any $f \in \mathscr{F}, f$ and $f^{\prime \prime}$ do not have any zero in $D$. Then $\left\{f^{\prime} \mid f: f \in \mathscr{F}\right\}$ is normal in $D$.

In the conclusion of Lemma $4, f^{\prime \prime}$ can be replaced by $f^{(k)}$ for any given $k \geq 2, k \in \mathbb{N}$. In fact, Hayman W. K. [8] raised a conjecture: 
Let $k \geq 2, k \in \mathbb{N}, f$ be a meromorphic function in $\mathbb{C}$ which satisfies $f \neq 0, f^{(k)} \neq 0$, then $f(z)=e^{a z+b}, a, b \in \mathbb{C}$ or $f(z)=1 /(a z+b)^{n}, n \in \mathbb{N}$.

Hayman [9], Clunie J [10], Mues E [11], Frank G [12] have studied the conjecture and Langley J. K. [13] has proved the conjecture at last. It is natural to ask whether there exists the normal rule to the conjecture. Schwick W. [14], Berweiler [1], Bergweiler and Langley [15] has given the normal rule. The case $k=2$ is enough here and has been proved by Bergweiler [1].

Lemma 5. Let $f$ be a meromorphic function, $D$ be an bounded domain and $c$ be constant in $\mathbb{C}$. If $f(z)-c$ has $l$ zeros in $D$ and $f^{\prime}(z)$ has $l-1$ zeros in $D$ which are all the zeros of $f(z)-c$. Then both $f(z)-c$ and $f^{\prime}(z)$ only have the same one discriminating zero in $D$.

Proof. Let $f(z)-c=\prod_{j=1}^{l}\left(z-z_{j}\right) g(z)=R(z) g(z)$, where $g(z)$ be a meromorphic function with no zero in $D$ and satisfies $g\left(z_{j}\right) \neq \infty(j=1, \ldots, l)$. Let $R(z)=\prod_{j=1}^{l}\left(z-z_{j}\right)$ with $z_{j} \subset D(j=$ $1, \ldots, l)$.

Because $f^{\prime}(z)$ has $l-1$ zeros in $D$ which are all the zeros of $f(z)-c$, then there exists $l-1$ points in $\left\{z_{j} \mid j=1, \ldots, l\right\}$ be zeros of $f^{\prime}(z)$ and with out loss of generality we may assume $f^{\prime}\left(z_{j}\right)=0(j=1, \ldots, l-1)$.

Because $f^{\prime}(z)=R^{\prime}(z) g(z)+R(z) g^{\prime}(z)$, we have

$$
R^{\prime}\left(z_{j}\right)=0(j=1, \ldots, l-1), R^{\prime}(z)=l \cdot \prod_{j=1}^{l-1}\left(z-z_{j}\right) \text { and } \frac{R^{\prime}(z)}{R(z)}=\frac{l}{z-z_{l}} .
$$

Then $R(z)=\left(z-z_{l}\right)^{l}$ and the proof is completed.

Lemma 6 ([8]). Let $f$ be a holomorphic function, if the spherical derivative of $f$ is bounded. Then the order of $f$ is at most 1.

\section{Proof of Theorem 1}

Proof. If the order of $f$ is at most 2, the conclusion is true due to the Lemma 3.

If the order of $f$ is greater than 2 , we apply Lemma 1 to obtain a sequence $\left\{\omega_{n}\right\}_{n=1}^{\infty}, \omega_{n} \rightarrow$ $\infty(n \rightarrow \infty)$ such that $f^{\#}\left(\omega_{n}\right) \rightarrow \infty,(n \rightarrow \infty)$. If there exists $M>0$ such that $M_{f}=\left\{f^{\prime}(z): z \in \mathbb{C}\right.$, $f(z)=0\} \subset \overline{D(0, M)} . \forall n \in \mathbb{N}$, let $f_{n}(z)=f\left(z+\omega_{n}\right)$. It is easy to apply Marty's theorem to know $\left\{f_{n}(z)\right\}_{n=1}^{\infty}$ is not normal at 0 . Because $M_{f_{n}} \subset M_{f} \subset \overline{D(0, M)}$, according to Lemma 2 there exists a sequence $\left\{z_{n}\right\}_{n=1}^{\infty}$, a sequence $\left\{\rho_{n}\right\}_{n=1}^{\infty}$ of positive real numbers and a non-constant finite order function $g(z)$ such that when $n \rightarrow \infty, z_{n} \rightarrow 0, \rho_{n} \rightarrow 0$ and

$$
\frac{f_{n}\left(z_{n}+\rho_{n} z\right)}{\rho_{n}}=\frac{f\left(\omega_{n}+z_{n}+\rho_{n} z\right)}{\rho_{n}} \stackrel{\text { loc. }}{\longrightarrow} g(z)
$$


in $\mathbb{C}$ where $g(z)$ satisfies $g^{\#}(z) \leq g^{\#}(0)=K+1$ for all $z \in \mathbb{C}$.

We first prove $g^{\prime}(z)$ cannot be constant. In fact, if $g(z)=A z+B(A, B \in \mathbb{C})$, then $g^{\#}(0)=$ $\frac{|A|}{1+|B|^{2}}=M+1$. However, it is obvious that $M_{g} \subset M_{f} \subset \overline{D(0, M)}$ and $|A| \leq M$. It contradicts $g^{\#}(0)=M+1$. Further, because $f^{\prime}(z) \neq 1(z \in \mathbb{C})$ and $g^{\prime}(z)$ not be constant, we apply Hurwitz's Theorem to have $g^{\prime}(z) \neq 1(z \in \mathbb{C})$. Notice that $M_{g} \subset \overline{D(0, M)}$ and $g(z)$ is finite order, by Lemma 3 we have $g(z)$ has the form $g(z)=z+a+\frac{b}{(z-c)^{l}}$ with $a, b, c \in \mathbb{C}, b \neq 0, l \in \mathbb{N}$.

$\forall n \in \mathbb{N}$, let $\tau_{n}=\omega_{n}+z_{n}$. There exists entire functions $F(z)$ and $H(z)$ such that $F(z)$ and $H(z)$ have no common non-trivial divisor and $z-f(z)=\frac{H(z)}{F(z)}$, then when $n$ is large enough, we have

$$
\begin{aligned}
\frac{f_{n}\left(z_{n}+\rho_{n} z\right)}{\rho_{n}}= & \frac{f\left(\tau_{n}+\rho_{n} z\right)}{\rho_{n}}=\frac{\tau_{n}+\rho_{n} a-\frac{H\left(\tau_{n}+\rho_{n} z\right)}{F\left(\tau_{n}+\rho_{n} z\right)}}{\rho_{n}} \stackrel{\text { loc. }}{\longrightarrow} z+a+\frac{b}{(z-c)^{l}}(z \in \mathbb{C}), \\
& \frac{\tau_{n}-\rho_{n} a-\frac{H\left(\tau_{n}+\rho_{n} z\right)}{F\left(\tau_{n}+\rho_{n} z\right)}}{\rho_{n}} \stackrel{\text { loc. }}{\longrightarrow} \frac{b}{(z-c)^{l}} \quad(n \rightarrow \infty, z \in \mathbb{C}) .
\end{aligned}
$$

Further,

$$
\begin{aligned}
& \frac{F\left(\tau_{n}+\rho_{n} z\right)}{H\left(\tau_{n}+\rho_{n} z\right)} \stackrel{\text { loc. }}{\Longrightarrow} 0 \quad(n \rightarrow \infty, z \in \mathbb{C} \backslash\{c\}), \\
& \frac{\left(\tau_{n}-\rho_{n} a\right) F\left(\tau_{n}+\rho_{n} z\right)-H\left(\tau_{n}+\rho_{n} z\right)}{\rho_{n} F\left(\tau_{n}+\rho_{n} z\right)} \stackrel{\text { loc. }}{\Longrightarrow} \frac{b}{(z-c)^{l}} \quad(n \rightarrow \infty, z \in \mathbb{C}), \\
& \frac{\rho_{n} F\left(\tau_{n}+\rho_{n} z\right)}{\left(\tau_{n}-\rho_{n} a\right) F\left(\tau_{n}+\rho_{n} z\right)-H\left(\tau_{n}+\rho_{n} z\right)} \stackrel{\text { loc. }}{\Longrightarrow} \frac{(z-c)^{l}}{b} \quad(n \rightarrow \infty, z \in \mathbb{C}) .
\end{aligned}
$$

Take an bounded domain $D$ includ $c$, from (2) we see $\left(\tau_{n}-\rho_{n} a\right) F\left(\tau_{n}+\rho_{n} z\right)-H\left(\tau_{n}+\rho_{n} z\right)$ have no zero in $D$ when $n$ is large enough; considering (3) there exists some sufficiently small neighborhood $D_{c}$ of $c$ such that $\overline{D_{c}} \subset D$, thus $F\left(\tau_{n}+\rho_{n} z\right)$ have $l$ zeros in $D_{c}$ when $n$ is large enough.

We divide two cases for $l$ :

Case $1=1$. Let

$$
\begin{aligned}
& g_{n}(z)=\frac{\left(\tau_{n}-\rho_{n} a\right) F\left(\tau_{n}+\rho_{n} z\right)-H\left(\tau_{n}+\rho_{n} z\right)}{\left(\tau_{n}-\rho_{n} a\right) F\left(\tau_{n}+\rho_{n} z\right)}, \\
& g_{n}^{\prime}(z)=\frac{\rho_{n}\left\{F^{\prime}\left(\tau_{n}+\rho_{n} z\right) H\left(\tau_{n}+\rho_{n} z\right)-H^{\prime}\left(\tau_{n}+\rho_{n} z\right) F\left(\tau_{n}+\rho_{n} z\right)\right\}}{\left(\tau_{n}-\rho_{n} a\right) F^{2}\left(\tau_{n}+\rho_{n} z\right)} .
\end{aligned}
$$

(2) can be expressed as:

$$
\frac{g_{n}(z)\left(\tau_{n}-\rho_{n} a\right)}{\rho_{n}}=\frac{\left(\tau_{n}-\rho_{n} a\right) F\left(\tau_{n}+\rho_{n} z\right)-H\left(\tau_{n}+\rho_{n} z\right)}{\rho_{n} F\left(\tau_{n}+\rho_{n} z\right)} \stackrel{\text { loc. }}{\longrightarrow} \frac{b}{z-c}(z \in \mathbb{C}) .
$$


From (2) we have $g_{n}(z)$ have no zero in $D_{c}$ when $n$ is large enough.

Here we need to prove $g_{n}^{\prime \prime}(z)$ have no zero in $D_{c}$. The derivative of (2) is

$$
\frac{g_{n}^{\prime}(z)\left(\tau_{n}-\rho_{n} a\right)}{\rho_{n}} \stackrel{\text { loc. }}{\longrightarrow}-\frac{b}{(z-c)^{2}}(z \in \mathbb{C} \backslash\{c\}) .
$$

The derivative of (3) is

$$
d_{n}(z)=\frac{\rho_{n}^{2}\left\{F^{\prime}\left(\tau_{n}+\rho_{n} z\right) H\left(\tau_{n}+\rho_{n} z\right)-F\left(\tau_{n}+\rho_{n} z\right) H^{\prime}\left(\tau_{n}+\rho_{n} z\right)\right\}}{\left\{\left(\tau_{n}-\rho_{n} a\right) F\left(\tau_{n}+\rho_{n} z\right)-H\left(\tau_{n}+\rho_{n} z\right)\right\}^{2}} \stackrel{\text { loc. }}{\longrightarrow} \frac{1}{b}(z \in \mathbb{C}) .
$$

From (4), when $n$ is large enough, $\left(\tau_{n}-\rho_{n} a\right) F\left(\tau_{n}+\rho_{n} z\right)-H\left(\tau_{n}+\rho_{n} z\right)$ have no zero in any bounded domain. Therefore, we apply Hurwitz Theorem to have $F^{\prime}\left(\tau_{n}+\rho_{n} z\right) H\left(\tau_{n}+\rho_{n} z\right)-$ $F\left(\tau_{n}+\rho_{n} z\right) H^{\prime}\left(\tau_{n}+\rho_{n} z\right)$ have no zero too. Considering

$$
\frac{\rho_{n}}{g_{n}^{\prime}(z)\left(\tau_{n}-\rho_{n} a\right)} \stackrel{\text { loc. }}{\Longrightarrow}-\frac{(z-c)^{2}}{b}(z \in \mathbb{C} \backslash\{c\}),
$$

then take the closed circle $D_{r} \subset D_{c}$ with center $c$ and we have

$$
\frac{\rho_{n}}{g_{n}^{\prime}(z)\left(\tau_{n}-\rho_{n} a\right)} \rightrightarrows-\frac{(z-c)^{2}}{b} \quad\left(z \in D_{r}\right)
$$

Further according to the Maximum modulus principle and Montel's normal criterion we have

$$
\frac{\rho_{n}}{g_{n}^{\prime}(z)\left(\tau_{n}-\rho_{n} a\right)} \stackrel{\text { loc. }}{\longrightarrow}-\frac{(z-c)^{2}}{b} \quad(z \in \mathbb{C}) .
$$

According to it's derivative, since $\left\{\frac{\rho_{n}}{g_{n}^{\prime}(z)\left(\tau_{n}-\rho_{n} a\right)}\right\}_{n=1}^{\infty}$ are holomorphic in any bound domain in $\mathbb{C}$, we have

$$
\frac{g_{n}^{\prime \prime}(z)\left(\tau_{n}-\rho_{n} a\right)}{\left\{g_{n}^{\prime}(z)\right\}^{2} \rho_{n}} \stackrel{\text { loc. }}{\Longrightarrow}-\frac{2(z-c)}{b} \quad(z \in \mathbb{C}),
$$

which means $g_{n}^{\prime \prime}(z) /\left\{g_{n}^{\prime}(z)\right\}^{2}$ have 1 zero in $D_{c}$ when $n$ is large enough. On the other hand,

$$
\frac{g_{n}^{\prime \prime}(z)}{\left\{g_{n}^{\prime}(z)\right\}^{2}}=\frac{1}{F^{4}\left(\tau_{n}+\rho_{n} z\right)\left\{g_{n}^{\prime}(z)\right\}^{2}}\left\{g_{n}^{\prime \prime}(z) F^{3}\left(\tau_{n}+\rho_{n} z\right)\right\} F\left(\tau_{n}+\rho_{n} z\right) .
$$

From (4) we see when $n$ is large enough, $F^{\prime}\left(\tau_{n}+\rho_{n} z\right) H\left(\tau_{n}+\rho_{n} z\right)-F\left(\tau_{n}+\rho_{n} z\right) H^{\prime}\left(\tau_{n}+\rho_{n} z\right)$ have no zero in $D_{c}$. After simple calculation, we have $1 / F^{4}\left(\tau_{n}+\rho_{n} z\right)\left\{g_{n}^{\prime}(z)\right\}^{2}$ are analytic and have no zero in $D_{c}$ with $g_{n}^{\prime \prime}(z) F^{3}\left(\tau_{n}+\rho_{n} z\right)$ are entire functions. Because $F\left(\tau_{n}+\rho_{n} z\right)$ have 1 zero in $D_{c}, g_{n}^{\prime \prime}(z)$ have no zero in $D_{c}$.

We apply Lemma 4 to obtain that $\left\{g_{n}^{\prime}(z) / g_{n}(z)\right\}_{n=1}^{\infty}$ is normal in $D_{c}$ and according to the definition of normal family there exists a subsequence we still suppose $\left\{g_{n}^{\prime}(z) / g_{n}(z)\right\}_{n=1}^{\infty}$ and a function $G(z)$ meromorphic in $D_{c}$ such that

$$
\frac{g_{n}(z)}{g_{n}^{\prime}(z)}=\frac{F\left(\tau_{n}+\rho_{n} z\right)\left\{\left(\tau_{n}-\rho_{n} a\right) F\left(\tau_{n}+\rho_{n} z\right)-H\left(\tau_{n}+\rho_{n} z\right)\right\}}{\rho_{n}\left\{F^{\prime}\left(\tau_{n}+\rho_{n} z\right) H\left(\tau_{n}+\rho_{n} z\right)-F\left(\tau_{n}+\rho_{n} z\right) H^{\prime}\left(\tau_{n}+\rho_{n} z\right)\right\}} \stackrel{\text { loc. }}{\Longrightarrow} G(z)
$$


where $G(z)$ cannot always be $\infty$ due to $F\left(\tau_{n}+\rho_{n} z\right)$ have 1 zero in $D_{c}$.

From (4) we have

$$
\frac{\rho_{n}}{\left(\tau_{n}-\rho_{n} a\right) d_{n}(z)} \stackrel{\text { loc. }}{\Longrightarrow} 0 \quad\left(z \in D_{c}\right)
$$

(5)-(6) is

$$
t_{n}(z)=\frac{H\left(\tau_{n}+\rho_{n} z\right)\left\{\left(\tau_{n}-\rho_{n} a\right) F\left(\tau_{n}+\rho_{n} z\right)-H\left(\tau_{n}+\rho_{n} z\right)\right\}}{\left(\tau_{n}-\rho_{n} a\right) \rho_{n}\left\{F^{\prime}\left(\tau_{n}+\rho_{n} z\right) H\left(\tau_{n}+\rho_{n} z\right)-H^{\prime}\left(\tau_{n}+\rho_{n} z\right) F\left(\tau_{n}+\rho_{n} z\right)\right\}} \stackrel{\text { loc. }}{\longrightarrow} G(z),
$$

then we have

$$
\frac{1}{t_{n}(z)} \stackrel{\text { loc. }}{\Longrightarrow} \frac{1}{G(z)}\left(z \in D_{c}\right)
$$

From (5) we have

$$
\frac{g_{n}^{\prime}(z)}{g_{n}(z)} \stackrel{\text { loc. }}{\longrightarrow} \frac{1}{G(z)}\left(z \in D_{c}\right)
$$

(7) $-(8)$ is

$$
\frac{\left\{F^{\prime}\left(\tau_{n}+\rho_{n} z\right) H\left(\tau_{n}+\rho_{n} z\right)-H^{\prime}\left(\tau_{n}+\rho_{n} z\right) F\left(\tau_{n}+\rho_{n} z\right)\right\} \rho_{n}}{F\left(\tau_{n}+\rho_{n} z\right) H\left(\tau_{n}+\rho_{n} z\right)} \stackrel{\text { loc. }}{\Longrightarrow} 0 \quad\left(z \in D_{c}\right) .
$$

Because $F^{\prime}\left(\tau_{n}+\rho_{n} z\right) H\left(\tau_{n}+\rho_{n} z\right)-F\left(\tau_{n}+\rho_{n} z\right) H^{\prime}\left(\tau_{n}+\rho_{n} z\right)$ have no zero in $D_{c}$ when $n$ is large enough, therefore from (9) we can see $F\left(\tau_{n}+\rho_{n} z\right)$ have no zero in $D_{c}$. However, it contradicts $F\left(\tau_{n}+\rho_{n} z\right)$ have 1 zero in $D_{c}$.

Case $2 l \geq 2$. From (3) we have

$$
\frac{\rho_{n} \frac{F\left(\tau_{n}+\rho_{n} z\right)}{H\left(\tau_{n}+\rho_{n} z\right)}}{\left(\tau_{n}-\rho_{n} a\right) \frac{F\left(\tau_{n}+\rho_{n} z\right)}{H\left(\tau_{n}+\rho_{n} z\right)}-1} \stackrel{\text { loc. }}{\Longrightarrow} \frac{(z-c)^{l}}{b}(z \in \mathbb{C}) .
$$

According to (1) and (10) we have

$$
\frac{\left(\tau_{n}-\rho_{n} a\right) F\left(\tau_{n}+\rho_{n} z\right)}{H\left(\tau_{n}+\rho_{n} z\right)} \stackrel{\text { loc. }}{\longrightarrow} 1 \quad(n \rightarrow \infty, z \in \mathbb{C} \backslash\{c\}) .
$$

Here we discuss two subcases for (11):

Subcase 2.1 If $\left\{\frac{\left(\tau_{n}-\rho_{n} a\right) F\left(\tau_{n}+\rho_{n} z\right)}{H\left(\tau_{n}+\rho_{n} z\right)}\right\}_{n=1}^{\infty}$ is normal in $\mathbb{C}$, from (11) we have

$$
\begin{aligned}
& \frac{\left(\tau_{n}-\rho_{n} a\right) F\left(\tau_{n}+\rho_{n} z\right)}{H\left(\tau_{n}+\rho_{n} z\right)} \stackrel{\text { loc. }}{\Longrightarrow} 1 \quad(z \in \mathbb{C}) . \\
& \frac{\left(\tau_{n}-\rho_{n} a\right) F\left(\tau_{n}+\rho_{n} z\right)-H\left(\tau_{n}+\rho_{n} z\right)}{H\left(\tau_{n}+\rho_{n} z\right)} \stackrel{\text { loc. }}{\Longrightarrow} 0 \quad(z \in \mathbb{C}) .
\end{aligned}
$$

From (2), $\left(\tau_{n}-\rho_{n} a\right) F\left(\tau_{n}+\rho_{n} z\right)-H\left(\tau_{n}+\rho_{n} z\right)$ have no zero in $D_{c}$ when $n$ is large enough, so are $H\left(\tau_{n}+\rho_{n} z\right)$ due to (13). According to Hurwitz's Theorem and (12), $F\left(\tau_{n}+\rho_{n} z\right)$ have no zero in $D_{c}$, which contradicts $F\left(\tau_{n}+\rho_{n} z\right)$ have $l$ zeros in $D_{c}$. 
Subcase 2.2 If $\left\{\frac{\left(\tau_{n}-\rho_{n} a\right) F\left(\tau_{n}+\rho_{n} z\right)}{H\left(\tau_{n}+\rho_{n} z\right)}\right\}_{n=1}^{\infty}$ is not normal in $\mathbb{C}$. Let

$$
\varphi_{n}(z)=\frac{\left(\tau_{n}-\rho_{n} a\right) F\left(\tau_{n}+\rho_{n} z\right)-H\left(\tau_{n}+\rho_{n} z\right)}{H\left(\tau_{n}+\rho_{n} z\right)} .
$$

Then $\left\{\varphi_{n}(z)\right\}_{n=1}^{\infty}$ is not normal and

$$
\varphi_{n}^{\prime}(z)=\frac{\left(\tau_{n}-\rho_{n} a\right) \rho_{n}\left\{H^{\prime}\left(\tau_{n}+\rho_{n} z\right) F\left(\tau_{n}+\rho_{n} z\right)-F^{\prime}\left(\tau_{n}+\rho_{n} z\right) H\left(\tau_{n}+\rho_{n} z\right)\right\}}{H^{2}\left(\tau_{n}+\rho_{n} z\right)} .
$$

The derivative of (3) is

$$
\rho_{n}^{2} \cdot \frac{F^{\prime}\left(\tau_{n}+\rho_{n} z\right) H\left(\tau_{n}+\rho_{n} z\right)-H^{\prime}\left(\tau_{n}+\rho_{n} z\right) F\left(\tau_{n}+\rho_{n} z\right)}{\left\{\left(\tau_{n}-\rho_{n} a\right) F\left(\tau_{n}+\rho_{n} z\right)-H\left(\tau_{n}+\rho_{n} z\right)\right\}^{2}} \stackrel{\text { loc. }}{\Longrightarrow} \frac{(z-c)^{l-1} l}{b}(z \in \mathbb{C}) .
$$

When $n$ is large enough, $\left(\tau_{n}-\rho_{n} a\right) F\left(\tau_{n}+\rho_{n} z\right)-H\left(\tau_{n}+\rho_{n} z\right)$ have no zero in $D_{c}$ from (2); and then $F^{\prime}\left(\tau_{n}+\rho_{n} z\right) H\left(\tau_{n}+\rho_{n} z\right)-H^{\prime}\left(\tau_{n}+\rho_{n} z\right) F\left(\tau_{n}+\rho_{n} z\right)$ have $l-1$ zeros in $D_{c}$ from (14) and Hurwitz's Theorem .

When $n$ is large enough, we see $\varphi_{n}(z)$ have no zero in $D_{c}$ from (2). Because $\left\{\varphi_{n}(z)\right\}_{n=1}^{\infty}$ is not normal, we apply Lemma 2 to obtain $\left\{v_{n}\right\}_{n=1}^{\infty} \in \mathbb{C}$ and $\left\{\rho_{n}^{*}\right\}_{n=1}^{\infty}$ of positive real numbers and a non-constant finite order function $\psi(\xi)$ such that $v_{n} \rightarrow c, \rho_{n}^{*} \rightarrow 0$, and

$$
\psi_{n}(\xi)=\frac{\varphi_{n}\left(v_{n}+\rho_{n}^{*} \xi\right)}{\rho_{n}^{*}} \stackrel{\text { loc. }}{\longrightarrow} \psi(\xi)(n \rightarrow \infty, \xi \in \mathbb{C}) \text { with } \psi^{\#}(\xi) \leq \psi^{\#}(0)=1 .
$$

Here we assert $\psi(\xi)$ has no simple pole if it exists. Let $\xi_{0}$ be the zero of $\psi(\xi)$. Beacuse $\psi(\xi)$ cannot always be $\infty$, there exists closed $\operatorname{disc} \bar{D}\left(\xi_{0}, \delta\right)$ such that $1 / \psi_{(\xi)}$ and $1 / \psi_{n}(\xi)$ are holomorphic in $\bar{D}\left(\xi_{0}, \delta\right)$. Then $1 / \psi_{n}(\xi) \rightrightarrows 1 / \psi(\xi)$ uniformly in $\bar{D}\left(\xi_{0}, \delta\right)$ and so are $1 / \psi_{n}(\xi)+\rho_{n}^{*}$.

Notice that $1 / \psi_{n}(\xi)$ cannot be constant, there exists $\left\{\xi_{n}\right\}_{n=1}^{\infty}, \xi_{n} \rightarrow \xi_{0}(n \rightarrow \infty)$ such that

$$
\frac{1}{\psi_{n}\left(\xi_{n}\right)}+\rho_{n}^{*}=\frac{\rho_{n}^{*}}{\varphi_{n}\left(v_{n}+\rho_{n}^{*} \xi_{n}\right)}+\rho_{n}^{*}=0, \quad \varphi_{n}\left(v_{n}+\rho_{n}^{*} \xi_{n}\right)+1=0
$$

We shall later show that the discriminating zeros of $\varphi_{n}(z)+1$ in $D_{c}$ are all the zeros of $\varphi_{n}^{\prime}(z)$ in $D_{c}$ when $n$ is large enough. In fact, $(z-f(z))^{\prime}=\frac{H^{\prime}(z) F(z)-H(z) F^{\prime}(z)}{F^{2}(z)} \neq 0(z \in \mathbb{C})$ therefore the zeros of $H^{\prime}(z) F(z)-H(z) F^{\prime}(z)$ are all the zeros of $F(z)$ in $\mathbb{C}$. Further, the zeros of $\varphi_{n}^{\prime}(z)$ in $D_{c}$ are all the zeros of $\varphi_{n}(z)+1$ in $D_{c}$. From (14) we see $\varphi_{n}^{\prime}(z)$ have $l-1$ zeros in $D_{c}$ when $n$ is large enough and $\varphi_{n}(z)+1$ have the same $l$ zeros with $F\left(\tau_{n}+\rho_{n} z\right)$ in $D_{c}$. Then by Lemma 5 the conclusion is proved and we have

$$
\left.\left(\frac{1}{\psi(\xi)}\right)^{\prime}\right|_{\xi=\xi_{0}}=-\frac{\psi^{\prime}\left(\xi_{0}\right)}{\left\{\psi\left(\xi_{0}\right)\right\}^{2}}=-\lim _{n \rightarrow \infty} \frac{\psi_{n}^{\prime}\left(\xi_{n}\right)}{\left\{\psi_{n}\left(\xi_{n}\right)\right\}^{2}}=-\lim _{n \rightarrow \infty}\left\{\rho_{n}^{*}\right\}^{2} \cdot 0=0,
$$

which means $\psi(\xi)$ has to have multiple pole if it exists, the assert is proved. 
Notice $\frac{H^{\prime}(z) F(z)-H(z) F^{\prime}(z)}{F^{2}(z)} \neq 0(z \in \mathbb{C})$, then $H\left(\tau_{n}+\rho_{n} z\right)$ have no multiple zero in $\mathbb{C}$.

Considering $1 / \psi_{n}(\xi) \stackrel{\text { loc. }}{\Longrightarrow} 1 / \psi(\xi)(\xi \in \mathbb{C})$. Since $\left(\tau_{n}-\rho_{n} a\right) F\left(\tau_{n}+\rho_{n} z\right)-H\left(\tau_{n}+\rho_{n} z\right)$ have no zero in $D_{c}$ when $n$ is large enough, $1 / \psi_{n}(\xi)$ are analytic in $D_{c}$. By Hurwitz's Theorem, $\psi(\xi)$ has no multiple pole and with the assert above, $\psi(\xi)$ have no pole and be entire.

Notice that $\psi^{\#}(\xi) \leq \psi^{\#}(0)=1(\xi \in \mathbb{C})$. By Lemma 6 , the order of $\psi(\xi)$ is at most 1 . Since $\varphi_{n}(z)$ have no zero in $D_{c}$ when $n$ is large enough, then $\varphi_{n}\left(v_{n}+\rho_{n}^{*} \xi_{n}\right)$ have no zero in any bounded domain, from the above it follows that $\psi(\xi) \neq 0(\xi \in \mathbb{C})$ and $\psi(\xi)=e^{A \xi+B}(A, B \in \mathbb{C}, A \neq$ $0)$.

$$
\begin{aligned}
& 1 / \psi_{n}(\xi) \stackrel{\text { loc. }}{\Longrightarrow} 1 / \psi(\xi)(\xi \in \mathbb{C}) \text { is } \\
& \frac{\rho_{n}^{*} H\left(\tau_{n}+\rho_{n} v_{n}+\rho_{n} \rho_{n}^{*} \xi\right)}{\left(\tau_{n}-\rho_{n} a\right) F\left(\tau_{n}+\rho_{n} v_{n}+\rho_{n} \rho_{n}^{*} \xi\right)-H\left(\tau_{n}+\rho_{n} v_{n}+\rho_{n} \rho_{n}^{*} \xi\right)} \stackrel{\text { loc. }}{\longrightarrow} e^{-A \xi-B} \quad(\xi \in \mathbb{C}) . \\
& \text { Let } \eta_{n}(z)=\frac{F\left(\tau_{n}+\rho_{n} z\right) H^{\prime}\left(\tau_{n}+\rho_{n} z\right)-F^{\prime}\left(\tau_{n}+\rho_{n} z\right) H\left(\tau_{n}+\rho_{n} z\right)}{\left\{\left(\tau_{n}-\rho_{n} a\right) F\left(\tau_{n}+\rho_{n} z\right)-H\left(\tau_{n}+\rho_{n} z\right)\right\}^{2}}
\end{aligned}
$$

Then the derivative of (15) is

$$
\left(\rho_{n}^{*}\right)^{2} \rho_{n}\left(\tau_{n}-\rho_{n} a\right) \eta_{n}\left(v_{n}+\rho_{n}^{*} \xi\right) \stackrel{\text { loc. }}{\Longrightarrow}-A e^{-A \xi-B}(n \rightarrow \infty, \xi \in \mathbb{C}) .
$$

(14) can be expressed as:

$$
\rho_{n}^{2} \eta_{n}(z) \stackrel{\text { loc. }}{\longrightarrow} \frac{(z-c)^{l-1} l}{b}(n \rightarrow \infty, z \in \mathbb{C}) .
$$

$\forall n \in \mathbb{N}$, let $h_{n}(z)$ be the $l-1$ order derivative of $\eta_{n}(z)(z$ be defined in any bounded domain in $\mathbb{C}$ ), then the $l-1$ order derivative of (14) is

$$
\rho_{n}^{2} h_{n}(z) \stackrel{\text { loc. }}{\Longrightarrow} \frac{l !}{b} \quad(n \rightarrow \infty, z \in \mathbb{C}) .
$$

Then we have

$$
\rho_{n}^{l+1} h_{n}\left(v_{n}+\rho_{n}^{*} \xi\right) \stackrel{l o c .}{\Longrightarrow} \frac{l !}{b}(n \rightarrow \infty, \xi \in \mathbb{C}) .
$$

The $l-1$ order derivative of (16) is

$$
\left(\rho_{n}^{*}\right)^{l+1} \rho_{n}\left(\tau_{n}-\rho_{n} a\right) h_{n}\left(v_{n}+\rho_{n}^{*} \xi\right) \stackrel{l o c .}{\Longrightarrow}(-A)^{l} e^{-A \xi-B} \quad(n \rightarrow \infty, \xi \in \mathbb{C}),
$$

$(17)+(18)$ is

$$
\left\{\rho_{n}^{l}+\left(\tau_{n}-\rho_{n} a\right)\left(\rho_{n}^{*}\right)^{l+1}\right\} \rho_{n} h_{n}\left(v_{n}+\rho_{n}^{*} \xi\right) \stackrel{l o c .}{\Longrightarrow} \frac{l !}{b}+(-A)^{l} e^{-A \xi-B} \quad(n \rightarrow \infty, \xi \in \mathbb{C}) .
$$

It shows that $h_{n}\left(v_{n}+\rho_{n}^{*} \xi\right)$ have zeros in $\mathbb{C}$ when $n$ is large enough. However, it contradicts (17) by Hurwitz's Theorem and the proof is completed. 


\section{References}

[1] B. Walter, Normality and exceptional values of derivatives, Proc. Amer Math. Soc., 129(1999), 121-129.

[2] J. Chang, On meromorphic functions whose first derivatives have finitely many zeros, Bull. Lond. Math. Soc., 44 (2012), 703-715.

[3] A. L. Beiträge zur, Theorie der Meromorphen Funktionen, C. R. $7^{e}$ Congr. Math. Scand. Oslo, 19(1929), 84-88.

[4] X. Pang and L. Zalcman, Normal families and shared values, Bull. London Mtah. Soc., 32, 325-331.

[5] L. Zaclman, A heuristic principle in complex function theory, Amer. Math. (Monthly), 82(1975), 813-817.

[6] X. Pang, Bloch's principle and normal criterion, Sci. China, Ser. A, 32(1989), 782-791. MR91I:30031.

[7] X. Pang, On normal criterion of meromorphic functions, Sci. China, Ser. A, 33(1990), 521-527. MR92B:30041.

[8] J. Clunie and W. K. Hayman, The spherical derivative of integral and meromorphic functions, Comment Math. Helv., 40 (1966), 117-148.

[9] W. K. Hayman, Picard values of meromorphic functions and their derivatives, Ann. Math., 70 (1959), 9-42.

[10] J. Clunie, On integral and meromorphic functions, J. London Math. Soc., 37 (1962), 17-27.

[11] E. Mues, Über eine Vermutung von Hayman. Math. Z., 119(1972), 11-20.

[12] G. Frank, Eine Vermutung von Hayman über Nullstellen meromorpher Funktion, Math. Z., 149(1976), $29-36$.

[13] J. K. Langley, Proof of a conjecture of Hayman concering $f$ and f", J. London Math. Soc., 48(1993), 500-514.

[14] W. Schwick, Sharing values and normality, Arch Math., 59(1992), 50-54.

[15] W. Bergweiler and J. K. Langley, Nonvanishing derivatives and normal families, J. Anal. Math., 91 (2003), 353367.

Department of Mathematics, China University Mining \& Technology (Beijing), Beijing 100083, China.

E-mail: sdsxguofeng@163.com.cn

Department of Mathematics, Yunnan Normal University, Kunming 650500, China.

E-mail: liyuhua@ynnu.edu.cn 